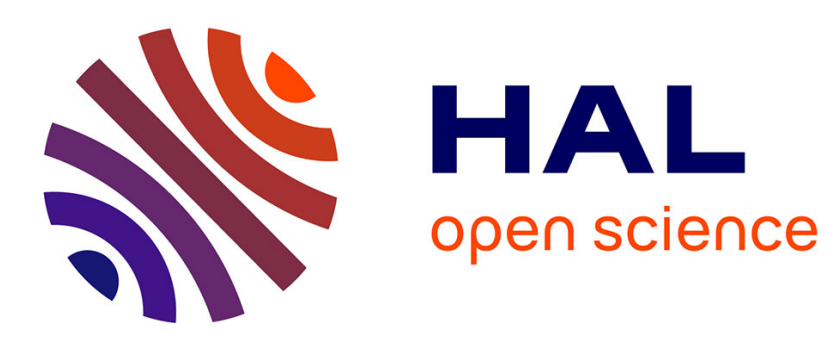

\title{
The surface/surface intersection problem by means of matrix based representations
}

\author{
Laurent Busé, Ba Thang Luu
}

\section{To cite this version:}

Laurent Busé, Ba Thang Luu. The surface/surface intersection problem by means of matrix based representations. Computer Aided Geometric Design, 2012, To appear. inria-00620947v1

\section{HAL Id: inria-00620947 \\ https://hal.inria.fr/inria-00620947v1}

Submitted on 9 Sep 2011 (v1), last revised 16 Jul 2012 (v2)

HAL is a multi-disciplinary open access archive for the deposit and dissemination of scientific research documents, whether they are published or not. The documents may come from teaching and research institutions in France or abroad, or from public or private research centers.
L'archive ouverte pluridisciplinaire HAL, est destinée au dépôt et à la diffusion de documents scientifiques de niveau recherche, publiés ou non, émanant des établissements d'enseignement et de recherche français ou étrangers, des laboratoires publics ou privés. 


\title{
The surface/surface intersection problem by means of matrix based representations
}

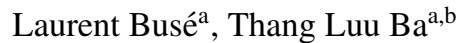 \\ ${ }^{a}$ INRIA, Galaad, 2004 route des Lucioles, 06902 Sophia Antipolis, France \\ ${ }^{b}$ Hanoi National University of Education, Department of Mathematics, 136 Xuan Thuy, Cau Giay, Hanoi, Vietnam
}

\begin{abstract}
Evaluating the intersection of two rational parameterized algebraic surfaces is an important problem in solid modeling. In this paper, we make use of some generalized matrix based representations of parameterized surfaces in order to represent the intersection curve of two such surfaces as the zero set of a matrix determinant. As a consequence, we extend to a dramatically larger class of rational parameterized surfaces, the applicability of a general approach to the surface/surface intersection problem due to J. Canny and D. Manocha. In this way, we obtain compact and efficient representations of intersection curves allowing to reduce some geometric operations on such curves to matrix operations using results from linear algebra.
\end{abstract}

\section{Introduction}

Computing the intersection between two parameterized algebraic surfaces is a fundamental task in Computer Aided Geometric Design. Several methods and approaches have been developed for that purpose. Some of them are based on the use of matrix representations of the objects because they allow to transform geometric operations on the intersection curve into matrix operations. This approach seems to have been first introduced by J. Canny and D. Manocha in their paper [16]. Roughly speaking, it consists in representing the implicit equation of one of the two surfaces as the determinant of a certain matrix, necessarily square. Then, instead of using this implicit equation, the matrix itself is used as a representation of this first parameterized surface and then a matrix representation of the intersection curve is easily obtained by substituting the implicit variables with the parameterization of the second surface.

As far as we know, this matrix based approach and all its successive improvements (see e.g. [13]), have only been developed by using square matrices for representing parameterized surfaces. These matrices are obtained either as resultant matrices, which is the case for instance in [16], or as matrices filled with some syzygies of a parameterization. This second class of matrices is known under the name of the moving quadrics method (see $[18,12,9])$. For these two classes of matrix representations, the limitation of their existence is basically that the parameterization of the surface has no base points (with the exception of the matrices given in [9]), that is to say is well defined over all its parameter space that could be a projective plane, which is the case for triangular Bézier surfaces, a product of projective lines, which is the case for tensor product surfaces, etc. This absence of base points is definitely a very strong constraint in the context of applications in solid modeling. It is hence the main limitation of applicability of the Canny-Manocha matrix based approach to the surface/surface intersection problem that is nevertheless an interesting comprise to provide an efficient, robust and accurate solution to this problem (see [16]). The goal of this paper is to overcome this limitation.

Recently, new matrix representations of parameterized algebraic surfaces have been introduced in $[11,7,6]$. These matrices are filled with syzygies of a parameterization in the same spirit as the moving quadrics method, but with the difference that they are non-square matrices. However they are still representation matrices because they are generically full rank matrices and their rank drop exactly on the surface (this is a direct generalization of a matrix determinant to non-square matrices). The main advantage of using these representation matrices is that they exist for a significantly larger class of parameterizations, in particular for most of parameterizations having base points (see $[11,7,8,6]$ for details). 
In this paper, we will introduce the use of these more general matrix representations in the context of the surface/surface intersection problem. By doing this, a new difficulty appears because we will have to manipulate pencils of bivariate matrices that are non-square, whereas in the classical Canny-Manocha approach the intersection curve is directly represented as a matrix determinant. It turns out that most techniques and algorithms that allow to determine the intersection curve with its exact topology need an implicit equation of this curve. Therefore, it is necessary to extract such an equation from a non-square matrix representation of this curve. For that purpose, instead of proceeding in a naive way by computing a greatest common divisor of several minors, we develop an algorithm based on matrix operations to extract from the non-square pencil of matrices all the information we need under the form of a pencil of square matrices. We will here follow the work of V. N. Kublanovskaya on what is called the $\Delta W-1$ decomposition algorithm [14].

The paper is organized as follows. In Section 2, we briefly define and review the matrix representations of a parametrized surface that we will use in the sequel. In Section 3, we deal with the surface/surface intersection problem. In particular, we show how to reduce the computation of the intersection curve of two parameterized surfaces to the determination of the spectrum of a bivariate pencil of matrices. Then, in Section 4 we propose a method, based on the $\Delta W-1$ decomposition of V. N. Kublanovskaya, that allows us to extract the regular part corresponding to the one-dimensional eigenvalues of such a bivariate pencil of matrices. The paper ends with some illustrative examples.

This work is a follow-up of a more general research program whose goal is to show that non-square matrix representations of algebraic curves and surfaces can be used in the place of square matrix representations with the advantage of being available for a dramatically larger class of parameterizations. Thus, in [2] the curve/surface intersection problem has been addressed and the space curve/space curve intersection problem is treated in [5]. As a natural continuation, we deal with the surface/surface intersection problem in this paper. This work is part of the $\mathrm{PhD}$ thesis of the second author [1].

For simplicity, throughout the paper the ground field will be an algebraically closed field, typically the field of complex numbers $\mathbb{C}$. However, mention that most of the results in Section 2, notably the matrix-based representations of surfaces we will introduce, could be given over an arbitrary infinite field.

\section{Matrix based implicit representations of parameterized surfaces}

Given a parameterized algebraic surface, we briefly recall from [11,7] how to build a matrix that represents this surface in a sense that we will make explicit. So suppose given a parameterization

$$
\begin{aligned}
\mathbb{P}_{\mathbb{C}}^{2} & \stackrel{\phi}{\rightarrow} \mathbb{P}_{\mathbb{C}}^{3} \\
(s: t: u) & \mapsto\left(f_{1}: f_{2}: f_{3}: f_{4}\right)(s, t, u)
\end{aligned}
$$

of a surface $\mathbf{S}$ such that $\operatorname{gcd}\left(f_{1}, \ldots, f_{4}\right) \in \mathbb{C} \backslash\{0\}$. Set $d:=\operatorname{deg}\left(f_{i}\right) \geq 1, i=1,2,3,4$ and denote by $x, y, z, w$ the homogeneous coordinates of the projective space $\mathbb{P}_{\mathbb{C}}^{3}$. The implicit equation of $\mathbf{S}$ is a homogeneous polynomial $S(x, y, z, w) \in \mathbb{C}[x, y, z, w]$ of smallest degree such that $S\left(f_{1}, f_{2}, f_{3}, f_{4}\right)=0$ (observe that it is defined up to multiplication by a nonzero element in $\mathbb{C})$. It is well known that the quantity $\operatorname{deg}(\mathbf{S}) \operatorname{deg}(\phi)$ is equal to $d^{2}$ minus the number of common roots of $f_{1}, f_{2}, f_{3}, f_{4}$ in $\mathbb{P}_{\mathbb{C}}^{2}$, that are called base points of the parameterization $\phi$, counted with suitable multiplicities (see for instance [11, Theorem 2.5] for more details). The notation $\operatorname{deg}(\phi)$ stands for the degree of the parameterization $\phi$ (co-restricted to $\mathbf{S}$ ) that, roughly speaking, measures the number of times the surface $\mathbf{S}$ is drawn by the parameterization $\phi$. The notation $\operatorname{deg}(\mathbf{S})$ stands for the degree of the surface $\mathbf{S}$ that is nothing but the degree of the implicit equation of $\mathbf{S}$.

For all non negative integer $v$, we build a matrix $\mathrm{M}(\phi)_{v}$ as follows. Consider the set $\mathcal{L}(\phi)_{v}$ of polynomials of the form

$$
a_{1}(s, t, u) x+a_{2}(s, t, u) y+a_{3}(s, t, u) z+a_{4}(s, t, u) w
$$

such that

- $a_{i}(s, t, u) \in \mathbb{C}[s, t, u]$ is homogeneous of degree $v$ for all $i=1, \ldots, 4$, 
- $\sum_{i=1}^{4} a_{i}(s, t, u) f_{i}(s, t, u) \equiv 0$ in $\mathbb{C}[s, t, u]$.

By definition, it is clear that $\mathcal{L}(\phi)_{v}$ is a $\mathbb{C}$-vector space and that a basis, say $L^{(1)}, \ldots, L^{\left(n_{v}\right)}$, of $\mathcal{L}(\phi)_{v}$ can be computed by solving a single linear system with indeterminates the coefficients of the polynomials $a_{i}(s, t, u), i=1,2,3,4$. The matrix $\mathrm{M}(\phi)_{v}$ is then by definition the matrix of coefficients of $L^{(1)}, \ldots, L^{\left(n_{v}\right)}$ as homogeneous polynomials of degree $v$ in the variables $s, t, u$. In other words, we have the equality of matrices:

$$
\left[\begin{array}{llll}
s^{v} & s^{v-1} t & \cdots & u^{v}
\end{array}\right] \mathrm{M}(\phi)_{v}=\left[\begin{array}{llll}
L^{(1)} & L^{(2)} & \cdots & L^{\left(n_{v}\right)}
\end{array}\right] .
$$

Notice that we have chosen for simplicity the monomial basis for the $\mathbb{C}$-vector space of homogeneous polynomials of degree $v$ in s,t,u. However, any other choice, for instance the Bernstein basis, can be made without affecting the result that we will state in the rest of this paper.

For all integer $v \geq 2 d-2$, the matrix $\mathrm{M}(\phi)_{v}$ is said to be a representation matrix of $\phi$ because it satisfies the following properties under the assumption that the base points of $\phi$, if any, form locally a complete intersection, that is to say are locally given by two equations (see [11]):

- The entries of $\mathrm{M}(\phi)_{v}$ are linear forms in $\mathbb{C}[x, y, z, w]$.

- The matrix $\mathrm{M}(\phi)_{v}$ has $\left(\begin{array}{c}v+2 \\ 2\end{array}\right)$ rows and possesses at least as much columns as rows.

- The rank of $\mathrm{M}(\phi)_{v}$ is $\left(\begin{array}{c}v+2 \\ 2\end{array}\right)$.

- When specializing $\mathrm{M}(\phi)_{v}$ at a given point $P \in \mathbb{P}_{\mathbb{C}}^{3}$, its rank drops if and only if $P$ belongs to $\mathbf{S}$.

- The greatest common divisor of the $\left(\begin{array}{c}v+2 \\ 2\end{array}\right)$-minors of $\mathrm{M}(\phi)_{v}$ is equal to the implicit equation of $\mathbf{S}$ raised to the power $\operatorname{deg}(\phi)$.

From a computational point of view, the matrix $\mathrm{M}(\phi)_{v}$ with the smallest possible value of $v$ has to be chosen. It is rarely a square matrix. Also, notice that the last property given above is never used for computations; our aim is to keep the matrix $\mathbf{M}(\phi)_{v}$ as an implicit representation of $\mathbf{S}$ in place of its implicit equation.

There are many results that lead to enlarge the above family of matrices and to make it available in other contexts. Since a detailed overview of these results is not the purpose of this paper, we just recall them shortly with appropriate references to the literature:

- The hypothesis on the base points of $\phi$ can be relaxed. If the base points are locally almost complete intersection, that is to say are locally given by three equations, then the above family of matrices can still be constructed and provide a matrix representation of the surface $\mathbf{S}$ plus a certain product of hyperplanes that can be described from the parameterization $\phi$. In addition, the bound $2 d-2$ for the integer $v$ can be decreased. See [7, 11].

- In our setting, $\phi$ parameterized what is called a triangular Bezier patch. It turns out that a similar family of matrices $\mathrm{M}(\phi)_{v}$ can be built for parameterizations of tensor product surfaces, and even for any parameterization with source a projective toric variety. See $[10,4,3]$.

- To build the matrices $\mathrm{M}(\phi)_{v}$ we used what is called moving planes, that is to say syzygies of the parameterization $\phi$. It is actually possible to build another family of matrices by taking into account moving quadrics, that is to say syzygies associated to the square of the ideal generated by the parameterization of $\phi$. In this way, we get a family containing smaller matrices whose entries are either linear or quadratic forms in $\mathbb{C}[x, y, z, w]$. In some sense, they generalize the matrices given in [12] and [9]. See [6].

\section{Representation of the intersection curve}

With the results presented in Section 2, we can generalize the Canny-Manocha's method for representing the intersection curve between two parameterized algebraic surfaces [16]. Indeed, the matrix based approach used by J. Canny and D. Manocha requires the existence of square matrix representations of parameterized algebraic surfaces. 
Hereafter, we will relax this limitation by using the matrix representations given in Section 2. Moreover, in Section 4 we will develop linear algebra techniques in order to obtain the intersection curve as a determinant matrix, exactly as in [16]. In this way, the output of our algorithm will be compatible with the determination of the exact topology of the intersection curve by means of several techniques, for instance [16] or more recently [17], that make use of an implicit equation.

Suppose given two parameterized algebraic surfaces $\mathbf{S}_{1}$ and $\mathbf{S}_{2}$. A standard problem in non linear computational geometry is to determine the set $\mathbf{S}_{1} \cap \mathbf{S}_{2} \subset \mathbb{P}_{\mathbb{C}}^{3}$ which is a curve in $\mathbb{P}_{\mathbb{C}}^{3}$ providing $\mathbf{S}_{1}$ and $\mathbf{S}_{2}$ are two distinct surfaces.

As we explained in Section 2, if the parameterization of $\mathbf{S}_{1}$ has locally complete intersection base points then one can build a representation matrix of $\mathbf{S}_{1}$ that we will denote by $M(x, y, z, w)$. Let

$$
\Psi: \mathbb{P}_{\mathbb{C}}^{2} \rightarrow \mathbb{P}_{\mathbb{C}}^{3}:(s: t: u) \mapsto(a(s, t, u): b(s, t, u): c(s, t, u): d(s, t, u))
$$

be a parameterization of $\mathbf{S}_{2}$ where $a(s, t, u), b(s, t, u), c(s, t, u), d(s, t, u)$ are homogeneous polynomials of the same degree and without common factor in $\mathbb{C}[s, t, u]$. By substituting in the representation matrix $M(x, y, z, w)$ the variables $x, y, z, w$ by the homogeneous polynomials $a(s, t, u), b(s, t, u), c(s, t, u), d(s, t, u)$ respectively, we get the matrix

$$
\mathbb{M}(s, t, u):=M(\Psi(s, t, u))=M(a(s, t, u), b(s, t, u), c(s, t, u), d(s, t, u)) .
$$

From the properties of the representation matrix $M(x, y, z, w)$, we known that $\mathbb{M}(s, t, u)$ has maximal rank $\rho$ ( $\rho$ is the number of rows of $\mathbb{M}$ and $\mathbb{M}$ has at least as much columns as rows). Moreover, for all point $\left(s_{0}: t_{0}: u_{0}\right) \in \mathbb{P}^{2}$ we have

$$
\operatorname{rank}\left(\mathbb{M}\left(s_{0}: t_{0}: u_{0}\right)\right)<\rho \text { if and only if }\left\{\begin{array}{l}
\Psi\left(s_{0}, t_{0}, u_{0}\right) \in \mathbf{S}_{1} \cap \mathbf{S}_{2} \\
\text { or } \\
\left(s_{0}: t_{0}: u_{0}\right) \text { is a base point of } \Psi .
\end{array}\right.
$$

Indeed, by property of the representation matrix $M(x, y, z, w)$, we know that the rank of $M$ drops at the point $\Psi\left(s_{0}, t_{0}, u_{0}\right)$, providing this later is well defined, if and only if $\Psi\left(s_{0}, t_{0}, u_{0}\right) \in \mathbf{S}_{1}$. If $\Psi\left(s_{0}, t_{0}, u_{0}\right)$ is not well defined, that is to say $\left(s_{0}: t_{0}: u_{0}\right)$ is a base point of $\Psi$, then the matrix $M\left(\Psi\left(s_{0}: t_{0}: u_{0}\right)\right)$ is equal to the null matrix since all the entries of $M$ are linear forms in $x, y, z, w$, an therefore its rank drops.

The equivalence (1) shows that the spectrum of the matrix $\mathbb{M}(s, t, u)$, that is to say the set

$$
\left\{\left(s_{0}: t_{0}: u_{0}\right) \in \mathbb{P}^{2} \text { such that } \operatorname{rank} \mathbb{M}\left(s_{0}: t_{0}: u_{0}\right)<\rho\right\},
$$

yields the intersection locus of $\mathbf{S}_{1} \cap \mathbf{S}_{2}$ plus the base points of the parameterization $\Psi$ of $\mathbf{S}_{2}$. The following result shows that the base points of $\Psi$ has not to be removed from the spectrum of $\mathbb{M}(s, t, u)$ that this later actually gives exactly the information we need to obtain the intersection locus $\mathbf{S}_{1} \cap \mathbf{S}_{2}$.

Theorem 1. The spectrum of the matrix $\mathbb{M}(s, t, u)$ is an algebraic curve in $\mathbb{P}^{2}$, that is to say is equal to the zero locus of a homogeneous polynomial in $\mathbb{C}[s, t, u]$. In particular, there is no isolated points in the spectrum of $\mathbb{M}(s, t, u)$.

Proof. By property of the representation matrix $M(x, y, z, w)$, we known that the ideal generated by the $\rho$-minors of $M(x, y, z, w)$, denoted $\operatorname{det}_{\rho} M(x, y, z, w)$, is supported on the hypersurface $\mathbf{S}_{1}$. In other words, the algebraic variety associated to $\operatorname{det}_{\rho} M(x, y, z, w)$ is exactly $\mathbf{S}_{1}$.

Now, since the substitution of $x, y, z, w$ by $\Psi(s, t, u)$ commutes with the formation of the determinantal ideals, that is to say

$$
\operatorname{det}_{\rho}(\mathbb{M}(s, t, u))=\operatorname{det}_{\rho}(M(\Psi(s, t, u)))=\left(\operatorname{det}_{\rho} M(x, y, z, w)\right)(\Psi(s, t, u)),
$$

we $\operatorname{deduce} \operatorname{det}_{\rho}(\mathbb{M}(s, t, u))$ is supported on the algebraic curve obtained by substituting $x, y, z, w$ by $\Psi(s, t, u)$ in an implicit equation of $\mathbf{S}_{1}$. We conclude the proof by noting that the spectrum of $\mathbb{M}(s, t, u)$ is by definition supported on the algebraic variety associated to the ideal $\operatorname{det}_{\rho}(\mathbb{M}(s, t, u))$.

Remark 2. If the parameterization of $\mathbf{S}_{1}$ has base points that are almost complete intersection, then the above results still hold but in the equivalence (1) and in the proof of Theorem 1, the surface $\mathbf{S}_{1}$ has to be replaced with $\left(\mathbf{S}_{1} \cup_{\mathfrak{p}} H_{\mathfrak{p}}\right)$ where $H_{\mathfrak{p}}$ is a hyperplane which is associated to a base point $\mathfrak{p}$ of the parameterization of $\mathbf{S}_{1}$ which is not a complete intersection. 
The algebraic curve $C \in \mathbb{P}^{2}$ in this theorem is a representation of the intersection set $\mathbf{S}_{1} \cap \mathbf{S}_{2}$ because the parameterization $\Psi$ induces a rational map from $C$ to $\mathbf{S}_{1} \cap \mathbf{S}_{2}$. Therefore, the computation of an equation of the curve $C$ allows to write down an explicit parameterization of the intersection locus $\mathbf{S}_{1} \cap \mathbf{S}_{2}$. In [16], such an equation is directly obtained as a determinant matrix because they restrict their study to the class of parameterized surfaces $\mathbf{S}_{1}$ that admit a square representation matrix. In our setting, since we handle a much more larger class of parameterized surfaces, we get non-square matrices. The purpose of the next section is therefore to describe an algorithm that allows to reduce these non-square matrices to square matrices by linear algebra methods. Then, in Section 5 we will state the resulting algorithm and illustrate it with some detailed examples. But before moving on, let us end this section with the following interesting geometric consequence of Theorem 1.

Corollary 3. For any parameterized surface $\mathbf{S}_{1}$ as above, the algebraic curve describing the spectrum of the matrix $\mathbb{M}(s, t, u)$ will always goes through the base points of the parameterization $\Psi$ of $\mathbf{S}_{2}$.

\section{Reduction of a bivariate pencil of matrices}

As a consequence of Theorem 1, if we use matrix representations to deal with the surface/surface intersection problem, we will end up at some point with a pencil of bivariate and non-square matrices that represents the intersection curve (after dehomogenization). Therefore, in order to be able to handle this intersection curve, for instance to determine its exact topology, it is necessary to extract a pencil of bivariate and square matrices that yields a matrix representation of the intersection curve as a matrix determinant. For that purpose, we develop an algorithm based on the remarkable work of V. N. Kublanovskaya [14].

\subsection{The spectrum of a bivariate polynomial matrix}

Let $M(s, t)$ be a matrix of size $m \times n$ depending on the two variables $\mathrm{s}$ and t. The spectrum of $M(s, t)$ is defined as the set

$$
\left\{\left(s_{0}, t_{0}\right) \in \mathbb{C} \times \mathbb{C}: \operatorname{rank}\left(M\left(s_{0}, t_{0}\right)\right)<\rho\right\}
$$

where $\rho:=\operatorname{rank} M(s, t)$. Denote by $M_{i_{1}, \ldots, i_{\rho}}^{j_{1}, \ldots, j_{\rho}}$ the matrix obtained from $M(s, t)$ by taking the $\rho$ rows of index $i_{1}, \ldots, i_{\rho}$, and the $\rho$ columns of index $j_{1}, \ldots, j_{\rho}$, where $1 \leq i_{1}<\ldots<i_{\rho} \leq m$ and $1 \leq j_{1}<\ldots<j_{\rho} \leq n$. The spectrum of $M(s, t)$ is exactly the set of common roots of all the algebraic equations

$$
\operatorname{det} M_{i_{1}, \ldots, i_{\rho}}^{j_{1}, \ldots, j_{\rho}}=0,1 \leq i_{1}<\ldots<i_{\rho} \leq m, 1 \leq j_{1}<\ldots<j_{\rho} \leq n .
$$

The one-dimensional and zero-dimensional algebraic components of the system of algebraic equations (2) determine respectively the one-dimensional and zero-dimensional eigenvalues of the matrix $M(s, t)$. We recall that the onedimensional eigenvalues (also called eigencurves) form the continuous part of the spectrum of $M(s, t)$ which can be described by an algebraic equation in the parameters $s$ and $t$. The zero-dimensional eigenvalues form the discrete part of the spectrum of $M(s, t)$.

\subsection{Linearization of a bivariate polynomial matrix}

Suppose given an $m \times n$-matrix $M(s, t)=\left(a_{i, j}(s, t)\right)$ with polynomial entries $a_{i, j}(s, t) \in \mathbb{C}[s, t]$. It can be equivalently written as a polynomial in $s$ whose coefficients are $m \times n$-matrices with entries in $\mathbb{C}[t]$. If $d=\max _{i, j}\left\{\operatorname{deg}_{s}\left(a_{i, j}(s, t)\right)\right\}$ then set

$$
M(s, t)=M_{d}(t) s^{d}+M_{d-1}(t) s^{d-1}+\ldots+M_{0}(t)
$$

where $M_{i}(t) \in \mathbb{C}[t]^{m \times n}$ for all $i=0, \ldots, d$.

Definition 4. The generalized companion matrices $A(t), B(t)$ of the matrix $M(s, t)$ are the matrices with coefficients in $\mathbb{C}[t]$ of size $((d-1) m+n) \times d m$ that are given by

$$
A(t)=\left(\begin{array}{ccccc}
0 & I_{m} & \ldots & \ldots & 0 \\
0 & 0 & I_{m} & \ldots & 0 \\
\vdots & \vdots & \vdots & \vdots & \vdots \\
0 & 0 & \ldots & \ldots & I_{m} \\
M_{0}^{t}(t) & M_{1}^{t}(t) & \ldots & \ldots & M_{d-1}^{t}(t)
\end{array}\right), B(t)=\left(\begin{array}{ccccc}
I_{m} & 0 & \ldots & \ldots & 0 \\
0 & I_{m} & 0 & \ldots & 0 \\
\vdots & \vdots & \vdots & \vdots & \vdots \\
0 & 0 & \ldots & I_{m} & 0 \\
0 & 0 & \ldots & \ldots & -M_{d}^{t}(t)
\end{array}\right),
$$


where $I_{m}$ stands for the identity matrix of size $m$ and $M_{i}^{t}(t)$ stands for the transpose of the matrix $M_{i}(t)$.

These companion matrices allows to linearize the polynomial matrix $M(s, t)$ in the sense that there exists two unimodular matrices $E(s, t)$ et $F(s, t)$ with coefficients in $\mathbb{C}[s, t]$ and of size $d m$ and $(d-1) m+n$ respectively, such that

$$
E(s, t)(A(t)-s B(t)) F(s, t)=\left(\begin{array}{c|c}
{ }^{t} M(s, t) & 0 \\
\hline 0 & I_{m(d-1)}
\end{array}\right) .
$$

In particular, a straightforward computation shows that for all $s \in \mathbb{C}$ and $v \in \mathbb{C}[t]^{m}$ we have

$$
M^{t}(s, t) v=0 \Leftrightarrow(A(t)-s B(t))\left(\begin{array}{c}
v \\
s v \\
\vdots \\
s^{d-1} v
\end{array}\right)=0 .
$$

It is important to notice that (3) implies that the computation of the spectrum of the polynomial matrix $M(s, t)$ can be reduced to the computation of the spectrum of the polynomial matrix $A(t)-s B(t)$ which has the advantage to be linear in the variable $s$.

\subsection{The $\Delta W-1$ decomposition}

Hereafter, we present an algorithm due to V. N. Kublanovskaya and which is called the $\Delta W-1$ decomposition. Given an univariate polynomial matrix $M(t)$, this decomposition transforms $M(t)$ into the form [ $\Delta(t), 0]$ by columns operations, where $\Delta(t)$ is a polynomial matrix of full column rank and 0 is a zero block-matrix. Notice that such a transformation is classical for matrices with coefficients in $\mathbb{C}$ by using LU-decomposition for instance (see e.g. [2] in a similar setting), but for a polynomial matrix this task is much more complicated.

In what follows, we give the $\Delta W-1$ decomposition which is due to V. N. Kublanovskaya [14]. Anyway, we will provide a detailed presentation and a sketch of proof because this algorithm is very hard to follow directly from [14].

Suppose given an univariate polynomial matrix $M(t)$ of size $m \times n$ and write it under the form

$$
\begin{aligned}
M(t) & =\left[M_{s, 1}, 0,0, \ldots, 0\right] t^{s}+\left[M_{s-1,1}, M_{s-1,2}, 0, \ldots, 0\right] t^{s-1}+\cdots \\
& +\left[M_{s-p+2,1}, \ldots, M_{s-p+2, p-1}, 0\right] t^{s-p+2}+\left[M_{s-p+1,1}, \cdots, M_{s-p+1, p-1}, M_{s-p+1, p}\right] t^{s-p+1} \\
& +\left[M_{s-p, 1}, \ldots, M_{s-p, p-1}, M_{s-p, p}\right] t^{s-p}+\cdots+\left[M_{0,1}, M_{0,2}, \ldots, M_{0, p}\right]
\end{aligned}
$$

where $1 \leq p \leq s+1$ and for each $j=1,2, \ldots, p$, the block matrices $M_{i, j}$ is of size $m \times t_{j}$ for all $i=0,1, \ldots, s$. We have $\sum_{j=1}^{p} t_{j}=n$ and the notation 0 stands for a zero block-matrix of appropriate size.

Lemma 5. Suppose given an univariate polynomial matrix $M(t)$ of size $m \times n$ and an integer $p$ such that $1 \leq p \leq s+1$ and (4) holds. If the matrix $M=\left[M_{s 1}, M_{s-1,2}, \ldots, M_{s-p+1, p}\right]$ is of full column rank then the matrix $M(t)$ is of full column rank.

Proof. Suppose that the matrix $M(t)$ is not of full column rank. Then, there exists a polynomial vector $F(t) \in \mathbb{C}[t]^{n \times 1}$, $F(t) \neq 0$ such that $M(t) F(t)=0$. Denoting by $k$ the maximum of the degree of the entries of $F(t)$, we can write

$$
F(t)=F_{k} t^{k}+F_{k-1} t^{k-1}+\ldots+F_{0}
$$

where $F_{i}=\left(F_{i, 1}, F_{i, 2}, \ldots, F_{i, n}\right)^{t} \in \mathbb{C}^{n \times 1}$, for all $i=0,1, \ldots, k$ and $F_{k} \neq 0$. From the equality

$$
\begin{aligned}
M(t) F(t)=0 & =\left(\left[M_{s, 1}, 0,0, \ldots, 0\right] F_{k}\right) t^{s+k}+\left(\left[M_{s-1,1}, M_{s-1,2}, 0, \ldots, 0\right] F_{k}+\left[M_{s, 1}, 0,0, \ldots, 0\right] F_{k-1}\right) t^{s+k-1} \\
& +\left(\left[M_{s, 1}, 0,0, \ldots, 0\right] F_{k-2}+\left[M_{s-1,1}, M_{s-1,2}, 0, \ldots, 0\right] F_{k-1}+\left[M_{s-2,1}, M_{s-2,2}, M_{s-2,3}, \ldots, 0\right] F_{k}\right) t^{s+k-2} \\
& +\cdots+\left[M_{0,1}, M_{0,2}, \ldots, M_{0, p}\right] F_{0}
\end{aligned}
$$


we deduce that the coefficients with respect to the variable $t$ of the matrix $M(t) F(t)$ are null matrices. Moreover, recall that the matrix $M=\left[M_{s, 1}, M_{s-1,2}, \ldots, M_{s-p+1, p}\right]$ is of full column rank by assumption. So, looking at the coefficient of $t^{s+k}$, we obtain that necessarily

$$
F_{k, 1}=F_{k, 2}=\ldots=F_{k, t_{1}}=0 .
$$

Looking now at the coefficient of $t^{s+k-1}$, we obtain that necessarily

$$
F_{k-1,1}=F_{k-1,2}=\ldots=F_{k-1, t_{1}}=0 \text { and } F_{k, t_{1}+1}=F_{k, t_{1}+2}=\ldots=F_{k, t_{1}+t_{2}}=0 .
$$

Continuing this way step by step, we finally arrive at the conclusion that $F_{k}$ has to be the null vector and hence get a contradiction.

\section{Corollary 6. Let}

$$
M(t)=t^{s} M_{s}+t^{s-1} M_{s-1}+\ldots+M_{0}
$$

be an univariate polynomial matrix of size $m \times n$. If $M_{s}$ is of full column rank then $M(t)$ is of full column rank.

Proof. Apply Lemma 5 where the decomposition (4) is taken with $p=1$.

We are now ready to describe the $\Delta W-1$ decomposition algorithm. We suppose given an univariate polynomial matrix $M(t)$ of size $m \times n$, of rank $\rho$ and of degree $s \geq 1$. We write it as

$$
M(t)=t^{s} M_{s}+t^{s-1} M_{s-1}+\ldots+M_{0}
$$

where $M_{0}, M_{1}, \ldots, M_{s}$ are matrices with entries in $\mathbb{C}$. The $\Delta W-1$ decomposition consists in computing two polynomial matrices $W(t)$ and $\Delta(t)$ of size $n \times n$ and $m \times \rho$ respectively, such that

$$
M(t) W(t)=[\Delta(t), 0]
$$

with the properties that $W(t)$ is unimodular and that $\Delta(t)$ if of full column rank and of degree less or equal to $s$. The notation 0 stands here for the null matrix of size $m \times(n-\rho)$. The following algorithm computes two sequences of polynomial matrices $M_{0}(t)=M(t), M_{1}(t), \ldots, M_{l}(t)$ and $W_{1}(t), \ldots, W_{l}(t)$ such that $M_{l}(t)=[\Delta(t), 0], W_{k}(t)$ is unimodular and $M_{k}(t)=M_{k-1}(t) W_{k}(t)$ for all $k=1,2, \ldots, l$.

Step 1. Construct an auxiliary matrix $N_{1}$ as follows.

(i) If all the columns of $M_{s}$ are nonzero then $N_{1}:=M_{s}$. Otherwise, $M_{s}$ contains some zero columns. By performing column permutations, we can transform $M_{s}$ into $M_{s}^{*}:=\left[\bar{M}_{s, 1}, 0\right]$ where $\bar{M}_{s, 1}$ is the nonzero columns of $M_{s}$ of size $m \times t_{1}$. This transformation is then applied to the matrices $M_{q}$ for all $q=0, \ldots, s-1$, which is transformed into $M_{q}^{*}:=\left[M_{q, 1}, M_{q, 2}\right]$, where $M_{q, 1}, M_{q, 2}$ are of size $m \times t_{1}$ and $m \times\left(n-t_{1}\right)$ respectively.

(ii) If all the columns of $M_{s-1,2}$ are nonzero, then

$$
N_{1}:=\left[\bar{M}_{s, 1}, M_{s-1,2}\right] .
$$

Otherwise, the columns of the matrices $M_{q, 2}$ for all $q=0, \ldots, s-1$, can be permuted to get $M_{s-1,2}^{*}:=\left[\bar{M}_{s-1,2}, 0\right]$ where $\bar{M}_{s-1,2}$ is the nonzero columns of $M_{s-1,2}$ of size $m \times t_{2}$ and $M_{q, 2}^{*}:=\left[M_{q, 2}, M_{q, 3}\right], q=0, \ldots, s-2$. The matrices $M_{q, 2}, M_{q, 3}$ are of size $m \times t_{2}$ and $m \times\left(n-t_{1}-t_{2}\right)$ respectively.

(iii) if all the columns of $M_{s-2,3}$ are nonzero, then

$$
N_{1}:=\left[\bar{M}_{s, 1}, \bar{M}_{s-1,2}, M_{s-2,3}\right]
$$

Otherwise, the above process will be repeated and it terminates after $p \leq s+1$ steps with a matrix

$$
N_{1}:=\left[\bar{M}_{s, 1}, \bar{M}_{s-1,2}, \bar{M}_{s-2,3}, \ldots, \bar{M}_{s-p+2, p-1}, M_{s-p+1, p}\right]
$$

built with block-matrices of size $m \times t_{1}, m \times t_{2}, \ldots, m \times t_{p-1}$ and $m \times\left(n-\delta_{p-1}\right)$ respectively, where $\delta_{k}=\sum_{i=1}^{k} t_{i}$. 
(iv) If $p<s+1$, then all the columns of $N_{1}$ are nonzero. If $p=s+1$, the last columns of $N_{1}$ may be zero columns. In this case, we can reduce the matrix $M(t)$ by deleting zero columns and thus get a polynomial matrix $M^{\prime}(t)$ of smaller size, say $m \times n_{1}, n_{1} \leq n$. We get

$$
N_{1}:=[\underbrace{\bar{M}_{s 1}}_{t_{1}}, \underbrace{\bar{M}_{s-1,2}}_{t_{2}}, \ldots, \underbrace{\bar{M}_{s-p+2, p-1}}_{t_{p-1}}, \underbrace{\bar{M}_{s-p+1, p}}_{t_{p}}]
$$

where $\sum_{i=1}^{p} t_{i}=n_{1}$ and all the columns of $N_{1}$ are nonzero columns.

Step 2. If $N_{1}$ is of full column rank, then $\Delta(t)=M^{\prime}(t)$ because $M^{\prime}(t)$ is of full column rank (Lemma 5). The algorithm stops here. Otherwise, find an $n_{1} \times\left(n_{1}-r_{1}\right)$ matrix $T_{1}$ whose columns form a basis of the right null space of the matrix $N_{1}\left(r_{1}:=\operatorname{rank} N_{1}\right)$

Step 3. The matrix $T_{1}$ is transformed into a lower triangular matrix $L_{1}$ of size $n_{1} \times h_{1}, h_{1} \geq n_{1}-r_{1}$, without permuting rows. The matrix $L_{1}$ is such that its columns contain a basis of the null space of the matrix $N_{1}$ and possibly some columns of the identity matrix. The process to get the matrix $L_{1}$ from $T_{1}$ can be described in the following way.

In the case the leading minor of size $n_{1}-r_{1}$ of the matrix $T_{1}$ is not equal to zero, we obtain $L_{1}$ of size $n_{1} \times\left(n_{1}-r_{1}\right)$ by performing a Gaussian elimination by columns. In the case the leading minor of size $n_{1}-r_{1}$ of matrix $T_{1}$ is equal to zero, we also perform a Gaussian elimination by columns and proceed as follows: if the first entry on the diagonal is nonzero then do nothing, otherwise insert a column on the left having a 1 at the first position and zero elsewhere. Then, if the second entry on the diagonal is non-zero do nothing, otherwise insert a column between the first and second column with a 1 at the second position and zero elsewhere. We continue like this and thus insert a column each time there is a zero element on the diagonal. In this way, we end up with a matrix $L_{1}$ of size $n_{1} \times h_{1}, h_{1} \geq n_{1}-r_{1}$, of the form

$$
L_{1}=\left(\begin{array}{cccc}
\ddots & 0 & \ldots & 0 \\
* & \ddots & 0 & 0 \\
\ldots & \ldots & \ddots & 0 \\
\ldots & \ldots & \ldots & \ddots \\
\ldots & \ldots & \ldots & \ldots \\
* & * & * & *
\end{array}\right)
$$

where all the elements on the diagonal are nonzero.

For the following steps, we will assume for simplicity that $L_{1}$ is of size $n_{1} \times\left(n_{1}-r_{1}\right)$. Notice that if $h_{1}>n_{1}-r_{1}$, we only have to replace hereafter the number $n_{1}-r_{1}$ with $h_{1}$ and the identity matrix $I_{r_{1}}$ with $I_{n_{1}-h_{1}}$.

The matrix $L_{1}$ can written in block-matrices as follows:

(i) If $\delta_{k-1} \leq n_{1}-r_{1}$ and $\delta_{k}>n_{1}-r_{1}$ for all $k \leq p-1$ then

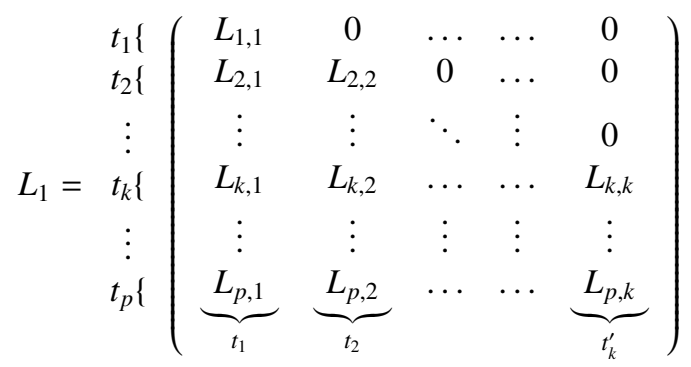

where $L_{i, i}, i=1,2, \ldots, k-1$ are nonsingular lower triangular matrices of size $t_{i} \times t_{i}$ and $L_{p, k}$ is a lower triangular matrix of size $t_{k} \times\left(n_{1}-r_{1}-\delta_{k-1}\right)$, so that $t_{k}^{\prime}=n_{1}-r_{1}-\delta_{k-1}$, and of full column rank. 
(ii) If $\delta_{k-1} \leq n_{1}-r_{1}$ and $\delta_{k}>n_{1}-r_{1}$ only for $k=p-1$ then

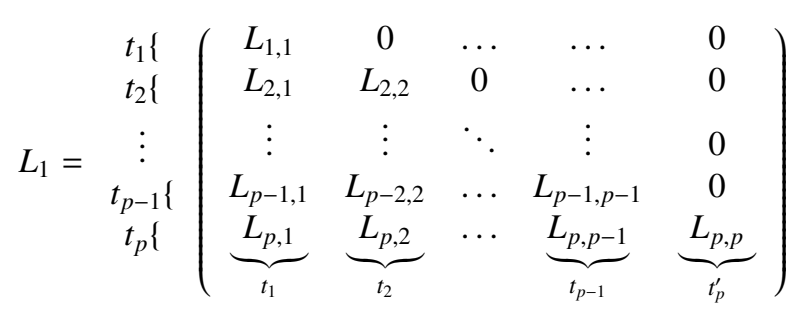

where $L_{i, i}, i=1,2, \ldots, p-1$ are nonsingular lower triangular matrices of size $t_{i} \times t_{i}$ and $L_{p p}$ is a lower triangular matrix of size $t_{p} \times\left(n_{1}-r_{1}-\delta_{p-1}\right)$, so that $t_{p}^{\prime}=n_{1}-r_{1}-\delta_{p-1}$, and of full column rank.

Step 4. Construct a left triangular unimodular $n_{1} \times n_{1}$ matrix $W^{(1)}(t)$ as follows

(i) If $L_{1}$ is of the form (6) then

$$
W^{(1)}(t)=\left(\begin{array}{ccccc}
L_{1,1} & 0 & \ldots & \ldots & 0 \\
t L_{2,1} & L_{2,2} & 0 & \ldots & 0 \\
\vdots & \vdots & \ddots & \vdots & 0 \\
t^{k-1} L_{k, 1} & t^{k-2} L_{k, 2} & \ldots & L_{k, k} & 0 \\
\vdots & \vdots & \vdots & \vdots & \vdots \\
t^{p-1} L_{p, 1} & t^{p-2} L_{p, 2} & \ldots & t^{p-k} L_{p, k} & I_{r_{1}}
\end{array}\right)
$$

where $I_{r_{1}}$ is the identity matrix of size $r_{1}$.

(ii) If $L_{1}$ is of the form (7) then

$$
W^{(1)}(t)=\left(\begin{array}{cccccc}
L_{1,1} & 0 & \ldots & \ldots & \ldots & 0 \\
t L_{2,1} & L_{2,2} & 0 & \ldots & \ldots & 0 \\
\vdots & \vdots & \ddots & \vdots & \vdots & 0 \\
\vdots & \vdots & \vdots & \vdots & \vdots & \vdots \\
t^{p-2} L_{p-1,1} & t^{p-3} L_{p-1,2} & \ldots & L_{p-1, p-1} & 0 & 0 \\
t^{p-1} L_{p, 1} & t^{p-2} L_{p, 2} & \ldots & t L_{p, p-1} & L_{p, p} & I_{r_{1}}
\end{array}\right)
$$

where $I_{r_{1}}$ is the identity matrix of size $r_{1}$.

Then, we define the unimodular polynomial matrix

$$
W_{1}(t)= \begin{cases}W^{(1)}(t) & \text { if } n=n_{1} \\ \operatorname{diagonal}\left\{W^{(1)}(t), I_{n-n_{1}}\right\} & \text { if } n>n_{1}\end{cases}
$$

Step 5. Compute the matrix

$$
M_{1}(t)=M_{0}(t) \Omega_{1} W_{1}(t)=M_{0}^{1}+t M_{1}^{1}+\ldots+t^{s_{1}} M_{s_{1}}^{1}
$$

where $s_{1} \leq s$ and $\Omega_{1}$ is a permutation matrix corresponding to the first step of this algorithm.

Step 6. Starting with $j=1$, we repeat the steps (1)-(5) for the matrix $M_{j}(t)$ until we find a matrix $M_{l+1}(t)$ of full column rank. Then, we get the $\Delta W-1$ decomposition

$$
M_{l}(t)=M(t) W(t)=[\Delta(t), 0]
$$

where $W(t)=\Omega \prod_{i=1}^{l} \Omega_{i} W_{i}(t)$ is an $n \times n$ unimodular polynomial matrix, $\Delta(t)$ is a polynomial matrix of full column rank and $\Omega$ is a permutation matrix corresponding to a last Gaussian elimination by columns. 
Sketch of proof of the $\Delta W-1$ decomposition algorithm. First, we see that the choice of the unimodular matrices $W_{i}(t)$ does not increase the degree of the matrix $M(t)$ under transformation. Thus, the degree of $\Delta(t)$ is not greater than the degree of $M(t)$.

Second, at each loop $k$ of the algorithm, the transformations eliminate $\left(n_{k}-r_{k}\right)>0$ vector coefficients at the greatest degree in $t$ of the columns of the polynomial matrix $M_{k-1}(t)$, this for all $k=1,2, \ldots, l+1$. Therefore, after a finite number of steps, the resulting matrix will have zero columns and its size can be reduced by deleting these zero columns, so that $n_{k}<n_{k-1}$. The matrices $M_{k}(t)$ and $M_{k-1}(t)$ have at least $r_{k}$ identical columns whose vector coefficients at the greatest degree are linearly independent. Thus, $r_{k+1} \geq r_{k}$. Therefore, the number $\left(n_{k}-r_{k}\right)$ decreases in a finite number of steps of the algorithm and finally, after the loop number $l$ we get $n_{l+1}-r_{l+1}=0$ and the algorithm stops here.

Finally, the matrix $\Delta(t)$ can be written under the form

$$
\begin{aligned}
\Delta(t) & =\left[\bar{\Delta}_{s, 1}, 0,0, \ldots, 0\right] t^{s}+\left[\Delta_{s-1,1}, \bar{\Delta}_{s-1,2}, 0, \ldots, 0\right] t^{s-1}+\ldots \\
& +\left[\Delta_{s-p+2,1}, \ldots, \bar{\Delta}_{s-p+2, p-1}, 0\right] t^{s-p+2}+\left[\Delta_{s-p+1,1}, \ldots, \Delta_{s-p+1, p-1}, \bar{\Delta}_{s-p+1, p}\right] t^{s-p+1} \\
& +\left[\Delta_{s-p, 1}, \ldots, \Delta_{s-p, p-1}, \Delta_{s-p, p}\right] t^{s-p}+\ldots+\left[\Delta_{0,1}, \Delta_{0,2}, \ldots, \Delta_{0, p}\right] .
\end{aligned}
$$

By construction, the matrix $N_{l+1}=\left[\bar{\Delta}_{s, 1}, \bar{\Delta}_{s-1,2}, \ldots, \bar{\Delta}_{s-p+1, p}\right]$ is of full column rank. By Lemma 5 , we deduce that $\Delta(t)$ is of full column rank.

Example 7. Let $M(t)$ be an univariate polynomial matrix

$$
M(t):=\left(\begin{array}{ccc}
2 t^{2}+3 t+1 & t+1 & 2 t^{2}+2 t \\
1 & 5 t^{2} & -5 t^{2}+1 \\
2 t+1 & 3 & 2 t-2 \\
t & t & 0
\end{array}\right)=M_{2} t^{2}+M_{1} t^{1}+M_{0} .
$$

We have

$$
M_{2}=\left(\begin{array}{ccc}
2 & 0 & 2 \\
0 & 5 & -5 \\
0 & 0 & 0 \\
0 & 0 & 0
\end{array}\right), M_{1}=\left(\begin{array}{lll}
3 & 1 & 2 \\
0 & 0 & 0 \\
2 & 0 & 2 \\
1 & 1 & 0
\end{array}\right), M_{0}=\left(\begin{array}{ccc}
1 & 1 & 0 \\
1 & 0 & 1 \\
1 & 3 & -2 \\
0 & 0 & 0
\end{array}\right) .
$$

Since all the columns of the matrix $M_{2}$ are nonzero columns, we set $N_{1}:=M_{2}$ and then the null space of $N_{1}$ is given by $L_{1}:=[1,-1,-1]^{t}$. So we form the matrix

$$
W^{(1)}(t)=\left(\begin{array}{ccc}
1 & 0 & 0 \\
-1 & 1 & 0 \\
-1 & 0 & 1
\end{array}\right)
$$

We obtain

$$
M_{1}(t)=M(t) W^{(1)}(t)=\left(\begin{array}{ccc}
0 & t+1 & 2 t^{2}+2 t \\
0 & 5 t^{2} & -5 t^{2}+1 \\
0 & 3 & 2 t-2 \\
0 & t & 0
\end{array}\right)
$$

The coefficient matrix of greatest degree of the matrix

$$
\Delta(t):=\left(\begin{array}{cc}
t+1 & 2 t^{2}+2 t \\
5 t^{2} & -5 t^{2}+1 \\
3 & 2 t-2 \\
t & 0
\end{array}\right)
$$

is given by

$$
\left(\begin{array}{cc}
0 & 2 \\
5 & -5 \\
0 & 0 \\
0 & 0
\end{array}\right)
$$

It is of full column rank and hence, by Corollary 6 , we deduce that the matrix $\Delta(t)$ is of full column rank. 
Example 8. Let $M(t)$ be the univariate polynomial matrix

$$
M(t):=\left(\begin{array}{cccc}
-t & 0 & t^{2} & 1+t^{3} \\
0 & -t & -1+t^{3} & -t^{2} \\
t^{2} & 1+t^{3} & t & 0
\end{array}\right)=M_{3} t^{3}+M_{2} t^{2}+M_{1} t+M_{0}
$$

where

$$
M_{3}=\left(\begin{array}{llll}
0 & 0 & 0 & 1 \\
0 & 0 & 1 & 0 \\
0 & 1 & 0 & 0
\end{array}\right), M_{2}=\left(\begin{array}{cccc}
0 & 0 & 1 & 0 \\
0 & 0 & 0 & -1 \\
1 & 0 & 0 & 0
\end{array}\right), M_{1}=\left(\begin{array}{cccc}
-1 & 0 & 0 & 0 \\
0 & -1 & 0 & 0 \\
0 & 0 & 1 & 0
\end{array}\right), M_{0}=\left(\begin{array}{cccc}
0 & 0 & 0 & 1 \\
0 & 0 & -1 & 0 \\
0 & 1 & 0 & 0
\end{array}\right) .
$$

Since the first column of the matrix $M_{3}$ is null, we permute the first column and the fourth column of $M_{3}$ and obtain

$$
M_{3}^{*}=\left(\begin{array}{llll}
1 & 0 & 0 & 0 \\
0 & 0 & 1 & 0 \\
0 & 1 & 0 & 0
\end{array}\right)
$$

and consequently the coefficient matrices $M_{2}, M_{1}, M_{0}$ are also permuted under the form

$$
M_{2}^{*}=\left(\begin{array}{cccc}
0 & 0 & 1 & 0 \\
-1 & 0 & 0 & 0 \\
0 & 0 & 0 & 1
\end{array}\right), M_{1}^{*}=\left(\begin{array}{cccc}
0 & 0 & 0 & -1 \\
0 & -1 & 0 & 0 \\
0 & 0 & 1 & 0
\end{array}\right), M_{0}^{*}=\left(\begin{array}{cccc}
1 & 0 & 0 & 0 \\
0 & 0 & -1 & 0 \\
0 & 1 & 0 & 0
\end{array}\right)
$$

Thus $N_{1}:=\left(\begin{array}{llll}1 & 0 & 0 & 0 \\ 0 & 0 & 1 & 0 \\ 0 & 1 & 0 & 1\end{array}\right)$. As the null space of $N_{1}$ is generated by $[0,1,0,-1]^{t}$, we get the matrix

$$
W^{(1)}(t):=\left(\begin{array}{cccc}
1 & 0 & 0 & 0 \\
0 & 1 & 0 & 0 \\
0 & 0 & 1 & 0 \\
0 & -t & 0 & 1
\end{array}\right)
$$

Denote by $P(m, n, k)$ the square matrix of size $k \times k$ obtained by permuting the columns number $m$ with the column number $n$. We have

$$
M_{1}(t):=M(t) P(1,4,4) W^{(1)}(t)=\left(\begin{array}{cccc}
1+t^{3} & t^{2} & t^{2} & -t \\
-t^{2} & -t & -1+t^{3} & 0 \\
0 & 1 & t & t^{2}
\end{array}\right)
$$

Repeating the above procedure to the polynomial matrix $M_{1}(t)$, we obtain the matrix

$$
N_{2}:=\left(\begin{array}{cccc}
1 & 0 & 1 & 0 \\
0 & 1 & 0 & 0 \\
0 & 0 & 0 & 1
\end{array}\right)
$$

and find that its null space is generated by $[1,0,-1,0]^{t}$. Thus,

$$
W^{(2)}(t):=\left(\begin{array}{cccc}
1 & 0 & 0 & 0 \\
0 & 1 & 0 & 0 \\
-t & 0 & 1 & 0 \\
0 & 0 & 0 & 1
\end{array}\right)
$$

and hence

$$
M_{2}(t):=M_{1}(t) P(2,3,4) W^{(2)}(t)=\left(\begin{array}{cccc}
t^{2} & 1 & t^{2} & -t \\
-1+t^{3} & 0 & -t & 0 \\
t & -t & 1 & t^{2}
\end{array}\right)
$$


Repeating the above procedure to the matrix $M_{2}(t)$, we obtain the matrix

$$
W^{(3)}(t):=\left(\begin{array}{cccc}
1 & 0 & 0 & 0 \\
0 & 1 & 0 & 0 \\
0 & 0 & 1 & 0 \\
0 & t & 0 & 1
\end{array}\right)
$$

and therefore

$$
M_{3}(t):=M_{2}(t) P(1,2,4) P(2,4,4) W^{(3)}(t):=\left(\begin{array}{cccc}
t^{2} & 0 & t^{2} & 1 \\
-1+t^{3} & 0 & -t & 0 \\
t & 0 & 1 & -t
\end{array}\right)
$$

The matrix

$$
\Delta(t):=\left(\begin{array}{ccc}
t^{2} & t^{2} & 1 \\
-1+t^{3} & -t & 0 \\
t & 1 & -t
\end{array}\right)=\left(\begin{array}{lll}
0 & 0 & 0 \\
1 & 0 & 0 \\
0 & 0 & 0
\end{array}\right) t^{3}+\left(\begin{array}{lll}
1 & 1 & 0 \\
0 & 0 & 0 \\
0 & 0 & 0
\end{array}\right) t^{2}+\left(\begin{array}{ccc}
0 & 0 & 0 \\
0 & -1 & 0 \\
1 & 0 & -1
\end{array}\right) t+\left(\begin{array}{ccc}
0 & 0 & 1 \\
-1 & 0 & 0 \\
0 & 1 & 0
\end{array}\right)
$$

is of full column rank because the matrix $\left(\begin{array}{ccc}0 & 1 & 0 \\ 1 & 0 & 0 \\ 0 & 0 & -1\end{array}\right)$ is of full column rank by Lemma 5 .

Remark 9. A Maple experimental program to perform the $\Delta W-1$ decomposition of an univariate polynomial matrix have been implemented and is freely available at the URL: http://www-sop.inria.fr/members/Luu.Ba_ Thang/.

4.4. The algorithm for extracting the regular part of a non square bivariate pencil of matrices

In the rest of this section, we provide an algorithm that extracts the continuous spectrum from a linearized bivariate pencil of matrices. We first state the main theorem and then give the algorithm.

Theorem $10([14,15])$. A $m \times n$-pencil of polynomial matrices $M(s, t)=A(t)-s B(t)$ is equivalent to a pencil of the following form

$$
\left(\begin{array}{ccc}
M_{1,1}(s, t) & 0 & 0 \\
M_{2,1}(s, t) & M_{2,2}(s, t) & 0 \\
M_{3,1}(s, t) & M_{3,2}(s, t) & M_{3,3}(s, t)
\end{array}\right)
$$

where

- the pencil $M_{2,2}(s, t)$ is a regular pencil that corresponds to the continuous spectrum of $M(s, t)$,

- the pencil $M_{1,1}(s, t)$ is of full row rank and determines the one-dimensional eigenvalues of the form $(0, t)$,

- the pencil $M_{3,3}(s, t)$ is of full column rank and determines the one-dimensional eigenvalues of the form $(\infty, t)$,

- the union of the discrete spectrum of the pencil $M_{1,1}(s, t)$ and $M_{3,3}(s, t)$ coincides with the discrete spectrum of $M(s, t)$.

We now describe an algorithm for constructing a pencil of the form (8). Set $\rho=\operatorname{rank} A(t), A_{1,1}(t)=A(t)$ and $B_{1,1}(t)=B(t)$.

Step 1 .

i) Transform $A_{1,1}(t)$ into the form $\left[\Delta_{0}(t), 0\right]$ by the $\Delta W-1$ decomposition, that means

$$
A_{1,1}(t) Q_{0}(t)=\left[\Delta_{0}(t), 0\right]
$$

where $Q_{0}(t)$ is an unimodular matrix. Then, compute

$$
B_{1,1}(t) Q_{0}(t)=[\underbrace{B_{1}(t)}_{\rho} \mid \underbrace{B_{2}(t)}_{n-\rho}] .
$$


ii) Determine an unimodular matrix $P_{0}(t)$ such that

$$
P_{0}(t) B_{2}(t)=\left(\frac{\bar{B}_{1,1}(t)}{0}\right)
$$

where $\bar{B}_{1,1}(t)$ has full row rank. At the end of ii), matrices $A(t)$ and $B(t)$ are represented under the form

$$
P_{0}(t) A_{1,1}(t) Q_{0}(t)=\left(\begin{array}{c|c}
A_{2,1}(t) & 0 \\
\hline A_{2,2}(t) & 0
\end{array}\right), \quad P_{0}(t) B_{1,1}(t) Q_{0}(t)=\left(\begin{array}{c|c}
B_{2,1}(t) & \bar{B}_{1,1}(t) \\
\hline B_{2,2}(t) & 0
\end{array}\right)
$$

where $\bar{B}_{1,1}(t)$ has full row rank and $\left(\frac{A_{2,1}(t)}{A_{2,2}(t)}\right)$ has full column rank. We have

$$
P_{0}(t)\left(A_{1,1}(t)-s B_{1,1}(t)\right) Q_{0}(t)=\left(\begin{array}{c|c}
A_{2,1}(t)-s B_{2,1}(t) & -s \bar{B}_{1,1}(t) \\
\hline A_{2,2}(t)-s B_{2,2}(t) & 0
\end{array}\right) .
$$

iii) By permuting rows, we obtain the matrix

$$
P P_{0}(t)\left(A_{1,1}(t)-s B_{1,1}(t)\right) Q_{0}(t)=\left(\begin{array}{c|c}
A_{2,2}(t)-s B_{2,2}(t) & 0 \\
\hline A_{2,1}(t)-s B_{2,1}(t) & -s \bar{B}_{1,1}(t)
\end{array}\right)
$$

where $\mathrm{P}$ is a permutation matrix.

Step 2. If the matrix $A_{2,2}(t)$ is not of full column rank, we repeat the Step 1 for the pencil $A_{2,2}(t)-s B_{2,2}(t)$ until, after $k$ repetitions, we get a matrix $A_{k+1, k+1}(t)$ which is of full column rank. Thus, we obtain the pencil

$$
P(t) M(s, t) Q(t)=\left(\begin{array}{c|c}
A_{k+1, k+1}(t)-s B_{k+1, k+1}(t) & 0 \\
\hline A_{k+1, k}(t)-s B_{k+1, k}(t) & M_{3,3}(t)
\end{array}\right)
$$

where $P(t), Q(t)$ are unimodular matrices.

If the pencil of the $m_{1} \times n_{1}$ matrix $A_{k+1, k+1}(t)-s B_{k+1, k+1}(t)$ is not a regular pencil, in particular it is not a square matrix and not of full row rank, then we repeat the above procedure to the transposed pencil $A_{k+1, k+1}^{t}(t)-s B_{k+1, k+1}^{t}(t)$. The steps consist in the following operations. Set $\tilde{A}_{1,1}(t)=A_{k+1, k+1}(t), \tilde{B}_{1,1}(t)=B_{k+1, k+1}(t)$.

i) Transform the transposed matrix $\tilde{B}_{1,1}^{t}(t)$ into the form $\left[\tilde{\Delta}_{0}(t), 0\right]$, that means

$$
\tilde{B}_{1,1}^{t}(t) \tilde{Q}_{0}(t)=[\underbrace{\tilde{\Delta}_{0}(t)}_{\tilde{\rho}} \mid \underbrace{0}_{m_{1}-\tilde{\rho}}]
$$

where $\tilde{Q}_{0}(t)$ is an unimodular matrix and $\tilde{\rho}=\operatorname{rank} \tilde{B}_{1,1}^{t}(t)$. Then, compute

$$
\tilde{Q}_{0}^{t}(t)\left(\tilde{A}_{1,1}(t)-s \tilde{B}_{1,1}(t)\right)=\left(\frac{\tilde{A}_{2}(t)-s \tilde{B}_{2}(t)}{\tilde{A}_{1}(t)-s .0}\right)
$$

where $\tilde{B}_{2}(t)=\tilde{\Delta}_{0}^{t}(t)$.

ii) Determine an unimodular matrix $\tilde{P}_{0}(t)$ such that

$$
\tilde{A}_{1}(t) \tilde{P}_{0}(t)=\left(\bar{A}_{1,1}(t) \mid 0\right)
$$

where $\bar{A}_{1,1}(t)$ is a $\tilde{\rho} \times \tilde{q}$ matrix of full column rank. At the end of ii), the pencil $\tilde{A}_{1,1}(t)-s \tilde{B}_{1,1}(t)$ is represented under the form

$$
Q_{0}^{t}(t)\left(\tilde{A}_{1,1}(t)-s \tilde{B}_{1,1}(t)\right) \tilde{P}_{0}(t)=\left(\begin{array}{c|c}
\tilde{A}_{2,1}(t)-s \tilde{B}_{2,1}(t) & \tilde{A}_{2,2}(t)-s \tilde{B}_{2,2}(t) \\
\hline \bar{A}_{1,1}(t) & 0
\end{array}\right) .
$$


iii) By permuting columns, we obtain the matrix

$$
Q_{0}^{t}(t)\left(\tilde{A}_{1,1}(t)-s \tilde{B}_{1,1}(t)\right) \tilde{P}_{0}(t) C=\left(\begin{array}{c|c}
\tilde{A}_{2,2}(t)-s \tilde{B}_{2,2}(t) & \tilde{A}_{2,1}(t)-s \tilde{B}_{2,1}(t) \\
\hline 0 & \bar{A}_{1,1}(t)
\end{array}\right)
$$

where $\mathrm{C}$ is a permutation matrix.

If the matrix $\tilde{B}_{2,2}(t)$ is not of full row rank, we repeat the above procedure to the pencil $\tilde{A}_{2,2}(t)-s \tilde{B}_{2,2}(t)$, say $l$ times, until the matrix $\tilde{B}_{l+1, l+1}(t)$ is of full row rank. In this way, we obtain the regular pencil $\tilde{A}_{l+1, l+1}(t)-$ $s \tilde{B}_{l+1, l+1}(t)$.

At the end, we obtain the following pencil which is equivalent to $M(s, t)$ :

$$
P(t) M(s, t) Q(t)=\left(\begin{array}{ccc}
M_{1,1}(s, t) & 0 & 0 \\
M_{2,1}(s, t) & M_{2,2}(s, t) & 0 \\
M_{3,1}(s, t) & M_{3,2}(s, t) & M_{3,3}(s, t)
\end{array}\right)
$$

where $P(t), Q(t)$ are unimodular matrices.

The pencil $M_{2,2}(t)=\tilde{A}_{l+1, l+1}(t)-s \tilde{B}_{l+1, l+1}(t)$ is regular. The pencil

$$
M_{1,1}(t)=\left(\begin{array}{cccc}
\bar{A}_{l, l}(t) & \tilde{A}_{l, l-1}(t)-s \tilde{B}_{l, l-1}(t) & \ldots & \tilde{A}_{l, 1}(t)-s \tilde{B}_{l, 1}(t) \\
0 & \bar{A}_{l-1, l-1}(t) & \ldots & \tilde{A}_{l-1,1}(t)-s \tilde{B}_{l-1,1}(t) \\
0 & 0 & \ddots & \vdots \\
0 & \ldots & 0 & \bar{A}_{1,1}(t)
\end{array}\right)
$$

is of full column rank and its spectrum does not contain any part of the continuous spectrum of $M(s, t)$, except for the one-dimensional eigenvalue of the form $(\infty, t)$. The pencil

$$
M_{3,3}(s, t)=\left(\begin{array}{ccccc}
-s \bar{B}_{k, k}(t) & 0 & 0 & \ldots & 0 \\
A_{k-1, k}(t)-s B_{k-1, k}(t) & -s \bar{B}_{k-1, k-1}(t) & 0 & \ldots & 0 \\
\vdots & \vdots & \ddots & \ldots & \vdots \\
A_{1, k}(t)-s B_{1, k}(t) & A_{1, k-1}(t)-s B_{1, k-1}(t) & A_{1, k-2}(t)-s B_{1, k-2}(t) & \ldots & -s \bar{B}_{1,1}(t)
\end{array}\right)
$$

is of full row rank and its spectrum does not contain any part of the continuous spectrum of $M(s, t)$, except for the one-dimensional eigenvalue of the form $(0, t)$.

Sketch of proof of Theorem 10

At the Step 1-i) of the algorithm, we can separate $Q_{0}(t)=\left[Q_{0,1}(t), Q_{0,2}(t)\right]$ where

$$
A(t) Q_{0,1}(t)=\Delta_{0}(t) \text { and } A(t) Q_{0,2}(t)=0 .
$$

Therefore, the columns of $Q_{0,2}(t)$ form a basis of right polynomial solutions of $A(t)$ and the finite spectrum of $\Delta_{0}(t)$ coincides with the finite spectrum of $A(t)$. At the Step 1-ii), the unimodular matrix $P_{0}(t)$ is written under the form $P_{0}(t)=\left(\frac{P_{0,1}(t)}{P_{0,2}(t)}\right)$ where

$$
P_{0,1}(t) B_{2}(t)=\bar{B}_{1,1}(t) \text { and } P_{0,2}(t) B_{2}(t)=0 .
$$

The rows of $P_{0,2}(t)$ form a basis of left polynomial solutions of $B_{2}(t)$ and the finite spectrum of $\bar{B}_{1,1}(t)$ coincides with the finite spectrum of $B_{2}(t)$. From

$$
P_{0}(t)(A(t)-s B(t)) Q_{0}(t)=\left(\begin{array}{c|c}
A_{2,1}(t)-s B_{2,1}(t) & -s \bar{B}_{1,1}(t) \\
\hline A_{2,2}(t)-s B_{2,2}(t) & 0
\end{array}\right),
$$

we deduce that the two subspaces $Q_{0,1}(t)$ and $P_{0,2}^{t}(t)$ form a pair of reducing subspaces (for instance, see [15, 19]) for the pencil $M(s, t)=A(t)-s B(t)$. By [15, Theorem 3.2], the union of the corresponding spectral characteristics of the 
blocks $-s \bar{B}_{1,1}(t)$ and $A_{2,2}(t)-s B_{2,2}(t)$ gives the whole spectrum and the right and left minimal indices with respect to $s$ of the original pencil $M(s, t)$. For more details, we refer the interested reader to $[14,15,19]$.

Before giving an illustrative example, mention that it is also possible to give algorithms to extract the discrete spectrum of a linearized pencil of polynomial matrices. However, as we will not need it to treat the surface/surface intersection problem, we refer the interested reader to [14, 15] or [1].

Example 11. Suppose given the bivariate linearized pencil of matrices $M(s, t)=A(s)-t B(s)$ where

$$
A(s)=\left(\begin{array}{cccc}
-s^{3} & -s^{2} & s^{2} & 0 \\
s & 0 & 1 & 0 \\
-s^{2}-s+1 & -s-1 & s & 1
\end{array}\right), B(s)=\left(\begin{array}{cccc}
-s^{2}-s & -s-1 & s+1 & 0 \\
s+1 & 1 & 1 & 0 \\
1+2 s & 2 & -1 & 1
\end{array}\right) .
$$

Applying the algorithm, we can compute unimodular polynomial matrices

$$
P_{0}(s):=\left(\begin{array}{ccc}
0 & -1 & 1 \\
0 & 2 s & 1-2 s \\
1 & 0 & 0
\end{array}\right), Q_{0}(s):=\left(\begin{array}{cccc}
0 & 0 & 0 & -1 \\
0 & 0 & 1 & 2 s \\
1 & 0 & 1 & s \\
0 & 1 & 0 & s+1
\end{array}\right)
$$

such that

$$
A_{1}(s):=P_{0}(s) A(s) Q_{0}(s)=\left(\begin{array}{ccc|c}
-1+s & 1 & -2 & 0 \\
\hline 3 s-2 s^{2} & 1-2 s & -1+4 s & 0 \\
s^{2} & 0 & 0 & 0
\end{array}\right)
$$

and

$$
B_{1}(s):=P_{0}(s) B(s) Q_{0}(s)=\left(\begin{array}{ccc|c}
-2 & 1 & -1 & 1 \\
\hline-1+4 s & 1-2 s & 1+2 s & 0 \\
s+1 & 0 & 0 & 0
\end{array}\right) .
$$

Therefore, we obtain the matrices

$$
A_{2}(s):=\left(\begin{array}{ccc}
3 s-2 s^{2} & 1-2 s & -1+4 s \\
s^{2} & 0 & 0
\end{array}\right), B_{2}(s):=\left(\begin{array}{ccc}
-1+4 s & 1-2 s & 1+2 s \\
s+1 & 0 & 0
\end{array}\right) .
$$

Applying the $\Delta W-1$ decomposition to the matrices $A_{2}(s)$ and $B_{2}(s)$, we compute the unimodular polynomial matrices

$$
P_{1}(s):=\left(\begin{array}{cc}
1 & 0 \\
0 & 1
\end{array}\right), Q_{1}(s):=\left(\begin{array}{ccc}
1 & 0 & 0 \\
0 & 1 & 2-8 s \\
0 & 1 / 2 & 2-4 s
\end{array}\right)
$$

such that

and

$$
A_{3}(s):=P_{1}(s) A_{2}(s) Q_{1}(s)=\left(\begin{array}{cc|c}
3 s-2 s^{2} & \frac{1}{2} & 0 \\
\hline s^{2} & 0 & 0
\end{array}\right)
$$

$$
B_{3}(s):=P_{1}(s) A_{2}(s) Q_{1}(s)=\left(\begin{array}{cc|c}
-1+4 s & \frac{3}{2}-s & 4-12 s+8 s^{2} \\
\hline s+1 & 0 & 0
\end{array}\right) .
$$

At last, we can transform the pencil of matrices $M(s, t)$ into the pencil

$$
\left(\begin{array}{c|ccc}
-1+s & 1 & -2 & 0 \\
3 s-2 s^{2} & \frac{1}{2} & 0 & 0 \\
\hline s^{2} & 0 & 0 & 0
\end{array}\right)-t\left(\begin{array}{cccc}
-2 & 1 & -1 & 1 \\
-1+4 s & \frac{3}{2}-s & 4-12 s+8 s^{2} & 0 \\
\hline s+1 & 0 & 0 & 0
\end{array}\right)
$$

where $M_{1}(s, t):=\left[s^{2}\right]-t[s+1]$ is the regular part of $M(s, t)$ and

$$
M_{2}(s, t):=\left(\begin{array}{ccc}
1 & -2 & 0 \\
\frac{1}{2} & 0 & 0
\end{array}\right)-t\left(\begin{array}{ccc}
1 & -1 & 1 \\
\frac{3}{2}-s & 4-12 s+8 s^{2} & 0
\end{array}\right)
$$

is of full row rank and determines the discrete spectrum of $M(s, t)$. Hence, the one-dimensional eigenvalue of $M(s, t)$ is given by the zero locus of $s^{2}-t(s+1)=0$ and we can easily see that the zero-dimensional eigenvalue of $M(s, t)$ is given by the pair $(s, t)=(1,1)$. 
Remark 12. A Maple experimental program corresponding to the above algorithm have been implemented and is freely available at the URL: http://www-sop.inria.fr/members/Luu.Ba_Thang/.

\section{Algorithm and illustrative examples}

Let us take again the setting and notation of Section 3. As a consequence of Theorem 1, the points in $\mathbf{S}_{1} \cap \mathbf{S}_{2}$ that are associated to points $(s: t: u) \in \mathbb{P}^{2}$ such that $u \neq 0$, are in correspondence with the $\operatorname{spectrum}$ of $\mathbb{M}(s, t, 1)$ and moreover, this spectrum is one-dimensional, it has no discrete component. Therefore, we can apply the results of Section 4 and we get the following algorithm:

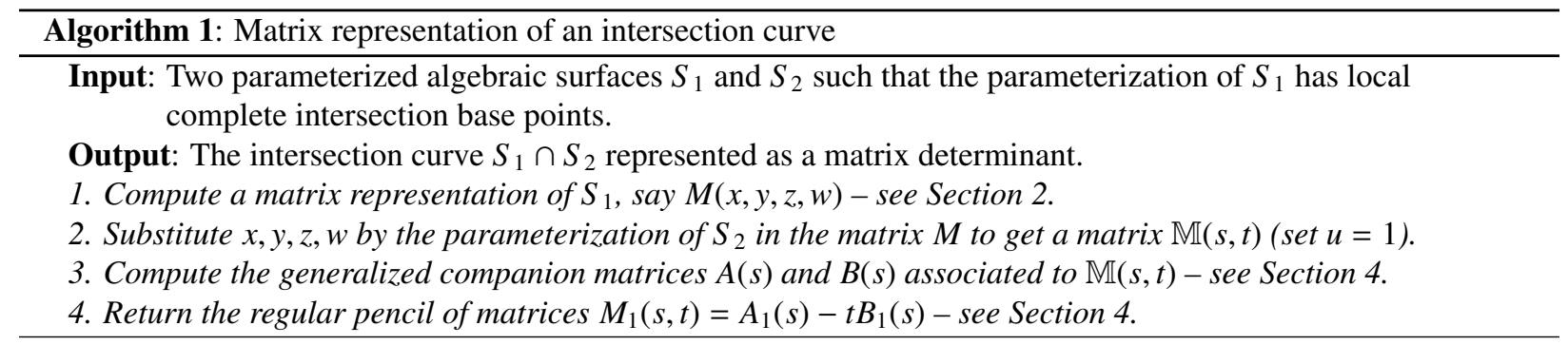

In comparison with [16], our algorithm give the same kind of result, that is to say a determinant matrix representation of the intersection curve, but the class of parameterizations of surfaces for which step 1 can be performed is here dramatically extended. We end this paper by giving some illustrative examples.

\subsection{Intersection of a sphere and a Steiner surface}

Let $\mathbf{S}_{1}$ be the sphere that we parameterized by

$$
\phi_{1}: \mathbb{P}^{2} \rightarrow \mathbb{P}^{3}:(s: t: u) \mapsto\left(s^{2}+t^{2}+u^{2}: 2 s u: 2 s t: s^{2}-t^{2}-u^{2}\right)
$$

and let $\mathbf{S}_{2}$ be the Steiner surface parametrized by

$$
\phi_{2}: \mathbb{P}^{2} \rightarrow \mathbb{P}^{3}:(s: t: u) \mapsto\left(s^{2}+t^{2}+u^{2}: t u: s u: s t\right) .
$$

The computation of a matrix representation of the sphere $\mathbf{S}_{1}$ yields

$$
\left(\begin{array}{cccc}
-y & 0 & z & x+w \\
0 & -y & -x+w & -z \\
z & x+w & y & 0
\end{array}\right)
$$

Therefore, the intersection locus $\mathbf{S}_{1} \cap \mathbf{S}_{2}$ is in correspondence with the spectrum of the polynomial matrix

$$
M(s, t, u)=\left(\begin{array}{cccc}
-t u & 0 & s u & s^{2}+t^{2}+u^{2}+s t \\
0 & -t u & -s^{2}-t^{2}-u^{2}+s t & -s u \\
s u & s^{2}+t^{2}+u^{2}+s t & t u & 0
\end{array}\right) .
$$

In order to apply our reduction algorithm on this matrix, we need to dehomogenize; for instance we choose here $u=1$ (notice that the case $u=0$ could also be treated as a curve/surface intersection problem as it is explained in [2]). So, we want to compute the spectrum of

$$
M(s, t)=\left(\begin{array}{cccc}
-t & 0 & s & s^{2}+t^{2}+1+s t \\
0 & -t & -s^{2}-t^{2}-1+s t & -s \\
s & s^{2}+t^{2}+1+s t & t & 0
\end{array}\right) .
$$


We have $M(s, t)=M_{2} t^{2}+M_{1} t+M_{0}$ and the generalized companion matrices associated to $M(s, t)$ are

$$
\begin{gathered}
A(s)=\left(\begin{array}{cc}
0 & I_{3} \\
M_{0}^{t} & M_{1}^{t}
\end{array}\right)=\left(\begin{array}{ccccccc}
0 & 0 & 0 & 1 & 0 & 0 \\
0 & 0 & 0 & 0 & 1 & 0 \\
0 & 0 & 0 & 0 & 0 & 1 \\
0 & 0 & s & -1 & 0 & 0 \\
0 & 0 & s^{2}+1 & 0 & -1 & s \\
s & -s^{2}-1 & 0 & 0 & s & 1 \\
s^{2}+1 & -s & 0 & s & 0 & 0
\end{array}\right), \\
B(s)=\left(\begin{array}{cccccc}
I_{3} & 0 \\
0 & -M_{3}^{t}
\end{array}\right)=\left(\begin{array}{cccccc}
1 & 0 & 0 & 0 & 0 & 0 \\
0 & 1 & 0 & 0 & 0 & 0 \\
0 & 0 & 1 & 0 & 0 & 0 \\
0 & 0 & 0 & 0 & 0 & 0 \\
0 & 0 & 0 & 0 & 0 & -1 \\
0 & 0 & 0 & 0 & 1 & 0 \\
0 & 0 & 0 & -1 & 0 & 0
\end{array}\right) .
\end{gathered}
$$

From the fact that the polynomial matrix $A(s)$ is of full column rank, we can apply the algorithm given in Section 4.4 for the pencil $A^{t}(s)-t B^{t}(s)$ and obtain the pencil $A_{1}(s)-t B_{1}(s)$ where

$$
A_{1}(s)=\left(\begin{array}{cccc|ccc}
1 & -s^{3} & 0 & 0 & 0 & 0 & 0 \\
0 & 0 & 0 & 1 & 0 & 0 & 0 \\
\hline-s^{2} & 1 & 0 & 0 & 0 & 0 & 0 \\
-s\left(s^{2}+1\right)+s^{2} & 0 & 1 & 0 & 0 & 0 & 0 \\
s+s^{2} & 0 & -1 & 1 & 0 & 0 & 0 \\
1-s^{2}+s^{3} & 1 & s & 0 & 0 & 0 & 0
\end{array}\right), B_{1}(s)=\left(\begin{array}{cccc|ccc}
0 & 0 & 1 & -s & 0 & s & 1 \\
0 & 0 & 0 & 0 & 0 & -1 & 0 \\
\hline s^{2} & 0 & -1 & 1 & 0 & 0 & 0 \\
s^{3} & 1 & -s & s & 0 & 0 & 0 \\
1 & 0 & 0 & 0 & 0 & 0 & 0 \\
-s^{2} & s & -1 & 0 & 0 & 0 & 0
\end{array}\right) .
$$

The regular part of the pencil $A^{t}(s)-t B^{t}(s)$ is then

$$
M_{1}(s, t)=\left(\begin{array}{cccc}
-s^{2} & 1 & 0 & 0 \\
-s\left(s^{2}+1\right)+s^{2} & 0 & 1 & 0 \\
s+s^{2} & 0 & -1 & 1 \\
1-s^{2}+s^{3} & 1 & s & 0
\end{array}\right)-t\left(\begin{array}{cccc}
s^{2} & 0 & -1 & 1 \\
s^{3} & 1 & -s & s \\
1 & 0 & 0 & 0 \\
-s^{2} & s & -1 & 0
\end{array}\right) .
$$

It gives a determinant matrix representation of the intersection curve. Thus, we could compute $\operatorname{det}\left(M_{1}(s, t)\right)=-\left(s^{2} t^{2}+\right.$ $s^{2}+s^{4}+t^{4}+t^{2}+1$ ) which is the equation of the curve that parameterizes $\mathbf{S}_{1} \cap \mathbf{S}_{2}$ through the regular map $\phi_{2}$.

Before moving to the next example, let us mention on this example how it is possible to determine if a given point in the projective space belongs to $\mathbf{S}_{1} \cap \mathbf{S}_{2}$. Suppose given a point $P \in \mathbb{P}^{3}$, for instance $P=(3: 2: 2: 1)$. We would like to check whether $P$ belongs to the intersection $\mathbf{S}_{1} \cap \mathbf{S}_{2}$. To do this, we can form matrix representations for both $\mathbf{S}_{1}$ and $\mathbf{S}_{2}$ and check their rank. Indeed, here we get

$$
M\left(\mathbf{S}_{1}\right):=\left(\begin{array}{cccc}
0 & y & x+w & z \\
-z & -x+w & -y & 0 \\
x+w & z & 0 & -y
\end{array}\right), M\left(\mathbf{S}_{2}\right):=\left(\begin{array}{ccccccccc}
0 & 0 & -y & w & z & 0 & 0 & -y & y \\
0 & -y & 0 & -x & y & 0 & -y & z & 0 \\
0 & 0 & 0 & w & 0 & 0 & z & 0 & y \\
-y & 0 & w & y & -x & -y & 0 & 0 & 0 \\
0 & w & 0 & 0 & 0 & z & 0 & 0 & -x \\
w & 0 & 0 & 0 & z & 0 & 0 & 0 & y
\end{array}\right),
$$

and evaluating the matrix $M\left(\mathbf{S}_{1}\right)$ and $M\left(\mathbf{S}_{2}\right)$ at the point $P$ we obtain the matrices

$$
M\left(\mathbf{S}_{1}\right)(P)=\left(\begin{array}{cccc}
0 & 2 & 4 & 2 \\
-2 & -2 & -2 & 0 \\
4 & 2 & 0 & -2
\end{array}\right), M\left(\mathbf{S}_{2}\right)(P)=\left(\begin{array}{ccccccccc}
0 & 0 & -2 & 1 & 2 & 0 & 0 & -2 & 2 \\
0 & -2 & 0 & -3 & 2 & 0 & -2 & 2 & 0 \\
0 & 0 & 0 & 1 & 0 & 0 & 2 & 0 & 2 \\
-2 & 0 & 1 & 2 & -3 & -2 & 0 & 0 & 0 \\
0 & 1 & 0 & 0 & 0 & 2 & 0 & 0 & -3 \\
1 & 0 & 0 & 0 & 2 & 0 & 0 & 0 & 2
\end{array}\right) .
$$


Since $M\left(\mathbf{S}_{1}\right)(P)$ has rank 2 and $M\left(\mathbf{S}_{2}\right)(P)$ has rank 6, we deduce that $P$ is a smooth point on the sphere $\mathbf{S}_{1}$ but $P$ does not belong to the Steiner surface $\mathbf{S}_{2}$, so $P \notin \mathbf{S}_{1} \cap \mathbf{S}_{2}$.

\subsection{Intersection of a sphere and a surface of degree 3}

As in the previous example, we start with the sphere $S_{1}$ parameterized by

$$
\phi_{1}: \mathbb{P}^{2} \rightarrow \mathbb{P}^{3}:(s: t: u) \mapsto\left(s^{2}+t^{2}+u^{2}: 2 s u: 2 s t: s^{2}-t^{2}-u^{2}\right)
$$

and admitting the matrix representation

$$
M(x, y, z, w):=\left(\begin{array}{cccc}
-y & 0 & z & x+w \\
0 & -y & -x+w & -z \\
z & x+w & y & 0
\end{array}\right)
$$

We want to determine the intersection between $S_{1}$ and the surface $S_{2}$ parameterized by

$$
\phi_{2}: \mathbb{P}^{2} \rightarrow \mathbb{P}^{3}:(s: t: u) \mapsto\left(s^{3}+t^{3}: s t u: s u^{2}+t u^{2}: u^{3}\right)
$$

$\mathbf{S}_{2}$ is a surface of degree 3. As in the previous example, to determine the intersection of $\mathbf{S}_{1}$ and $\mathbf{S}_{2}$ we will compute the spectrum of the polynomial matrix

$$
M(s, t, u)=\left(\begin{array}{cccc}
-s t u & 0 & s u^{2}+t u^{2} & s^{3}+t^{3}+u^{3} \\
0 & -s t u & -s^{3}-t^{3}+u^{3} & -s u^{2}-t u^{2} \\
s u^{2}+t u^{2} & s^{3}+t^{3}+u^{3} & s t & 0
\end{array}\right) .
$$

By dehomogenizing with respect to the variable $u$, we consider

$$
M(s, t)=\left(\begin{array}{cccc}
-s t & 0 & s+t & s^{3}+t^{3}+1 \\
0 & -s t & -s^{3}-t^{3}+1 & -s-t \\
s+t & s^{3}+t^{3}+1 & s t & 0
\end{array}\right) .
$$

We have $M(s, t)=M_{3} t^{3}+M_{2} t^{2}+M_{1} t+M_{0}$ and the generalized companion matrices of $M(s, t)$ are

$$
\begin{aligned}
& A(s)=\left(\begin{array}{ccc}
0 & I_{3} & 0 \\
0 & 0 & I_{3} \\
M_{0}^{t} & M_{1}^{t} & M_{2}
\end{array}\right)=\left(\begin{array}{ccccccccc}
0 & 0 & 0 & 1 & 0 & 0 & 0 & 0 & 0 \\
0 & 0 & 0 & 0 & 1 & 0 & 0 & 0 & 0 \\
0 & 0 & 0 & 0 & 0 & 1 & 0 & 0 & 0 \\
0 & 0 & 0 & 0 & 0 & 0 & 1 & 0 & 0 \\
0 & 0 & 0 & 0 & 0 & 0 & 0 & 1 & 0 \\
0 & 0 & 0 & 0 & 0 & 0 & 0 & 0 & 1 \\
0 & 0 & s & -s & 0 & 1 & 0 & 0 & 0 \\
0 & 0 & s^{3}+1 & 0 & -s & 0 & 0 & 0 & 0 \\
s & -s^{3}+1 & 0 & 1 & 0 & s & 0 & 0 & 0 \\
s^{3}+1 & -s & 0 & 0 & -1 & 0 & 0 & 0 & 0
\end{array}\right), \\
& B(s)=\left(\begin{array}{ccc}
I_{3} & 0 & 0 \\
0 & I_{3} & 0 \\
0 & 0 & -M_{3}^{t}
\end{array}\right)=\left(\begin{array}{ccccccccc}
1 & 0 & 0 & 0 & 0 & 0 & 0 & 0 & 0 \\
0 & 1 & 0 & 0 & 0 & 0 & 0 & 0 & 0 \\
0 & 0 & 1 & 0 & 0 & 0 & 0 & 0 & 0 \\
0 & 0 & 0 & 1 & 0 & 0 & 0 & 0 & 0 \\
0 & 0 & 0 & 0 & 1 & 0 & 0 & 0 & 0 \\
0 & 0 & 0 & 0 & 0 & 1 & 0 & 0 & 0 \\
0 & 0 & 0 & 0 & 0 & 0 & 0 & 0 & 0 \\
0 & 0 & 0 & 0 & 0 & 0 & 0 & 0 & -1 \\
0 & 0 & 0 & 0 & 0 & 0 & 0 & 1 & 0 \\
0 & 0 & 0 & 0 & 0 & 0 & -1 & 0 & 0
\end{array}\right)
\end{aligned}
$$


Applying the algorithm given in Section 4.4 for the pencil $A^{t}(s)-t B^{t}(s)$ and obtain its regular part $M_{1}(s, t)=A_{1}(s)-$ $t B_{1}(s)$ where

$$
A_{1}(s)=\left(\begin{array}{cccccc}
1 & 0 & s & 0 & 1 & 0 \\
-s^{3}+1 & 0 & 1 & 0 & 0 & 0 \\
-s^{3}+1 & 0 & 0 & -s & 0 & 0 \\
2 s & 0 & 0 & 1 & s & 0 \\
0 & 0 & 0 & 0 & 0 & 1 \\
0 & 1 & 0 & -1 & 0 & 0
\end{array}\right), B_{1}(s)=\left(\begin{array}{cccccc}
0 & 0 & 1 & 0 & 0 & 0 \\
0 & 1 & 0 & -1 & 0 & 0 \\
s^{3} & 1 & 0 & 0 & 0 & 0 \\
-s^{2} & 0 & s & 0 & 1 & 0 \\
1 & 0 & 0 & 0 & 0 & 0 \\
0 & 0 & 0 & 0 & 0 & 1
\end{array}\right)
$$

We get a curve of degree 6 , as expected since it is the result on the intersection of a surface of degree 2 and a surface of degree 3. We could compute $\operatorname{det}\left(M_{1}(s, t)\right)=-s^{6}-2 s^{3} t^{3}+t^{2} s^{2}+s^{2}+2 s t-t^{6}+t^{2}+1$ that is the equation of the curve $C$ (see Figure 1) that parameterizes $\mathbf{S}_{1} \cap \mathbf{S}_{2}$ (see Figure 2) through the regular map $\phi_{2}$. Notice that there are many methods to draw the intersection curve $\mathbf{S}_{1} \cap \mathbf{S}_{2}$ from the parametric curve $C$ through the regular map $\phi_{2}$; see for example [17].

Figure 1: The plane curve $C$ corresponding with $\mathbf{S}_{1} \cap \mathbf{S}_{2}$

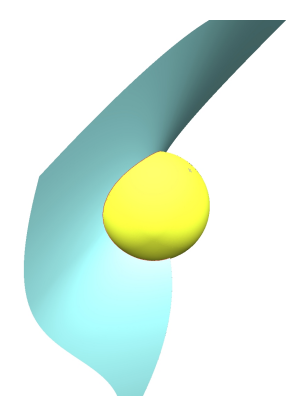

(a)

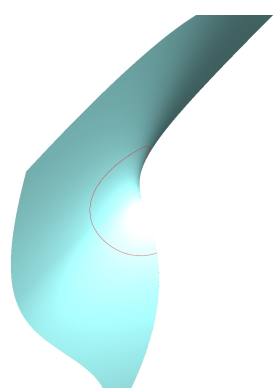

(b) (c)

Figure 2: Intersection of the sphere $\mathbf{S}_{1}$ and the surface $\mathbf{S}_{2}$

\section{References}

[1] T. Luu Ba. Matrix-based implicit representations of algebraic curves and surfaces and applications. PhD thesis, University of Nice Sophia Anitpolis, July 2011.

[2] T. Luu Ba, L. Busé, and B. Mourrain. Curve/surface intersection problem by means of matrix representation. In SNC'09: Proceedings of the International Conference on Symbolic Numeric Computation. Kyoto, Japan.ACM, NewYork, USA, pages 71-78, August 2009.

[3] Nicolás Botbol. Implicitization of rational maps. PhD thesis, University of Buenos Aires and the University of Pierre et Marie Curie, Paris 6, September 2010.

[4] Nicolás Botbol, Marc Dohm, and Alicia Dickenstein. Matrix representations for toric parametrizations. Computer Aided Geometric Design, 7:757-771, 2009.

[5] L. Busé and T. Luu Ba. Matrix-based implicit representations of algebraic curves and applications. Computer Aided Geometric Design, 27(9):681-699, 2010. 
[6] L. Busé, M. Chardin, and with appendix of J. Oesterlé Aron Simis. Elimination and nonlinear equations of rees algebras. Journal of Algebra, 324(3):1314-1333, 2010

[7] Laurent Busé and Marc Chardin. Implicitizing rational hypersurfaces using approximation complexes. J. Symbolic Comput., 40(4-5):1150$1168,2005$.

[8] Laurent Busé, Marc Chardin, and Jean-Pierre Jouanolou. Torsion of the symmetric algebra and implicitization. Proceedings of the American Mathematical Society, 137(06):1855-1865., February 2009.

[9] Laurent Busé, David Cox, and Carlos D'Andrea. Implicitization of surfaces in $\mathbb{P}^{3}$ in the presence of base points. J. Algebra Appl., 2(2):189214, 2003.

[10] Laurent Busé and Marc Dohm. Implicitization of bihomogeneous parametrizations of algebraic surfaces via linear syzygies. In ISSAC 2007, pages 69-76. ACM, New York, 2007.

[11] Laurent Busé and Jean-Pierre Jouanolou. On the closed image of a rational map and the implicitization problem. J. Algebra, 265(1):312-357, 2003.

[12] David Cox, Ronald Goldman, and Ming Zhang. On the validity of implicitization by moving quadrics of rational surfaces with no base points. J. Symbolic Comput., 29(3):419-440, 2000.

[13] Mario Fioravanti, Laureano Gonzalez-Vega, and Ioana Necula. Computing the intersection of two ruled surfaces by using a new algebraic approach. Journal of Symbolic Computation, 41(11):1187-1205, November 2006.

[14] V.N. Kublanovskaya. Methods and algorithm of solving spectral problems for polynomial matrices and rational matrix. Journal of Mathematical Sciences, 96(3):3085-3287, 1999.

[15] V.N. Kublanovskaya and V.B. Khazanov. Spectral problems for pencils of polynomial matrices. methods and algorithms v. Journal of Mathematical Sciences, 79(3):1048-1076, 1996.

[16] Dinesh Manocha and John Canny. A new approach for surface intersection. In Proceedings of the first ACM symposium on Solid modeling foundations and CAD/CAM applications, pages 209-219, Austin, Texas, United States, 1991. ACM.

[17] A. Mantzaflaris and B. Mourrain. A subdivision approach to planar semi -algebraic sets. In Proceedings of the 6th international Conference GMP, Castro Urdiales, Spain, pages 104-123, June 2010.

[18] T.W. Sederberg and F. Chen. Implicitization using moving curves and surfaces. In Proceedings of SIGGRAPH, volume 29, pages 301-308, 1995.

[19] G. W. Stewart. On the sensitivity of the eigenvalue problems $a x=\lambda b x$. SIAM J. Numer. Anal. 9, 4:669-689, 1972. 